\title{
Real impact of surgical robotic system for precision surgery of parotidectomy: retroauricular parotidectomy using da Vinci surgical system
}

\author{
Young Min Park, Da Hee Kim, Min Seok Kang, Jae-Yol Lim, Se-Heon Kim, Eun Chang Choi, \\ Yoon Woo Koh \\ Department of Otorhinolaryngology, Yonsei University College of Medicine, Seoul, Korea \\ Contributions: (I) Conception and design: YM Park, YW Koh; (II) Administrative support: DH Kim, MS Kang; (III) Provision of study materials or \\ patients: YM Park, YW Koh; (IV) Collection and assembly of data: YM Park, YW Koh; (V) Data analysis and interpretation: JY Lim, SH Kim, EC \\ Choi; (VI) Manuscript writing: All authors; (VII) Final approval of manuscript: All authors. \\ Correspondence to: Yoon Woo Koh, MD, PhD. Professor, Department of Otorhinolaryngology, Yonsei University College of Medicine, 50-1 Yonsei- \\ ro, Seodaemun-gu, Seoul 03722, Korea. Email: ywkohent@yuhs.ac.
}

\begin{abstract}
Background: We performed robotic parotidectomy with or without robotic neck dissection via a retroauricular approach in patients with parotid benign and malignant tumors and analyzed treatment outcomes of the patients to evaluate the safety and feasibility of robotic parotidectomy.

Methods: Between January 2017 and July 2018, 53 patients received robotic parotidectomy with/without robotic neck dissection through a remote access retroauricular incision without a preauricular incision.

Results: All operations were successfully performed in all patients without significant perioperative complications or tumor spillage. Tumors were located in the superficial lobe of the parotid gland in 40 patients, and the remaining 13 tumors were located in the deep lobe of the parotid gland. Postoperative pathologic examination revealed benign tumor in 32 patients and malignant tumors in 18 patients. The mean operation time was 226 minutes in patients who underwent only parotidectomy and 375 minutes in patients who underwent parotidectomy with robotic neck dissection. The average amount of bleeding was $23 \mathrm{~mL}$, and the amount of drainage after operation averaged $171 \mathrm{~mL}$. The average length of hospital stay was 6 days. Postoperative complications were limited to transient facial paralysis in three patients, all of which resolved within 1 month. All patients were satisfied with their cosmetic results at 6 months after operation.

Conclusions: Robotic parotidectomy with/without robotic neck dissection through a retroauricular approach was a feasible and safe technique in patients with parotid benign and malignant tumor. Specifically, we found it to be helpful in young patients with malignant parotid tumors who should receive cervical lymphadenectomy and parotidectomy, because it does not leave a visible scar on the face or neck. In the future, long-term follow-up will be necessary to validate its oncologic safety and functional outcomes.
\end{abstract}

Keywords: Robotic parotidectomy; retroauricular approach; parotid tumor; parotid cancer

Submitted Sep 03, 2019. Accepted for publication Jan 04, 2020.

doi: 10.21037 /gs.2020.01.05

View this article at: http://dx.doi.org/10.21037/gs.2020.01.05

\section{Introduction}

Recently, in the field of head and neck surgery, there have been many studies on remote access surgery using endoscopic equipment or robotic surgical systems to avoid visible scars on the face or neck. With the introduction of robotic surgical system, various operative techniques have been developed for thyroid surgery or other neck surgeries, including transaxillary, transoral, and retroauricular approaches (1-3). We first described robotic assisted neck dissection through a modified facelift or retroauricular 
Table 1 Patient demographics of all patients

\begin{tabular}{lccc}
\hline & Parotidectomy only $(\mathrm{n}=36)$ & $\begin{array}{c}\text { Parotidectomy with robotic neck } \\
\text { dissection }(\mathrm{n}=16)\end{array}$ & $\begin{array}{c}\text { Parotidectomy with thyroid } \\
\text { lobectomy }(\mathrm{n}=1)\end{array}$ \\
\hline $\begin{array}{l}\text { Mean age, y } \\
\text { Gender }\end{array}$ & 41 & 40 & 36 \\
Male & 29 & 6 & 0 \\
Female & 17 & 10 & 1 \\
Tumor location & & & 11 \\
Superficial lobe & 28 & 5 & 0 \\
Deep lobe & 8 & & 1 \\
Histologic type & 4 & 14 & 1 \\
Malignant tumor & 32 & 2 & 0 \\
Benign disease & 4 & \\
\hline
\end{tabular}

incision in 2013 (4,5). We have applied the same surgical technique to patients with parotid cancer. The da Vinci surgical system was implemented through a modified facelift incision for both parotidectomy and cervical lymphadenectomy of levels II-V. In these patents, cosmetic outcomes were excellent, resulting in high levels of patient satisfaction reported on a five-point scale. Our new operative technique has also shown comparable oncologic outcomes and superior cosmetic results (6).

In head and neck surgery, the advantages of the robotic surgical system are that it provides remote access to the surgical site while avoiding a visible scar on the face and neck. With increased experience, we have come to realize that a $10 \times$ magnified view of the operative field and bimanual technique using two robotic arms allowed surgeons to perform fine tissue manipulation and precise surgery compared to conventional surgery. Usually, the facial nerve is identified and distinguished from the blood vessels by naked eye during parotic surgery; however, distinguishing between the facial nerve and surrounding vessels is not always easy, especially in parotid surgery. If the parotidectomy is performed using a robotic surgical system, facial nerve identification and preservation can be easier due to three dimensional magnified visualization of operative field and fine tissue dissection by using three articulated robotic arms. On 10× magnified view of surgical field, the nerve can be easily distinguished from the vessels and preserved without an injury.

Since then, we have performed robotic parotidectomy with robotic neck dissection through only a retroauricular incision without preauricular extension using a da Vinci Xi system. In this study, we analyzed the treatment outcomes of patients who received our procedure to evaluate the safety and feasibility of robotic parotidectomy. To date, this study is the first to report on robotic parotidectomy using a da Vinci system.

\section{Methods}

\section{Patients}

We retrospectively analyzed the medical records of 53 patients who underwent robotic retroauricular parotidectomy at Severance Hospital from January 2017 to November 2018. This study was approved by the Institutional Review Board of Yonsei University. Before operation, all patients were given an explanation about the advantages and disadvantages of robotic parotidectomy and other surgical treatment methods available. All patients actively participated in selecting the treatment method. Of the 53 patients, 36 underwent only robotic parotidectomy, 16 underwent robotic parotidectomy combined with robot-assisted neck dissection (selective neck dissection of level I-III), and one underwent robotic parotidectomy combined with robotic thyroid lobectomy (Table 1). In cases in which preoperative FNAB and imaging studies suggested benign disease, we usually did not perform a neck dissection. Meanwhile, in cases in which malignant tumor was suspected on the results of preoperative FNAB, we considered a simultaneous neck dissection, especially when suspected lymph nodes were observed in 

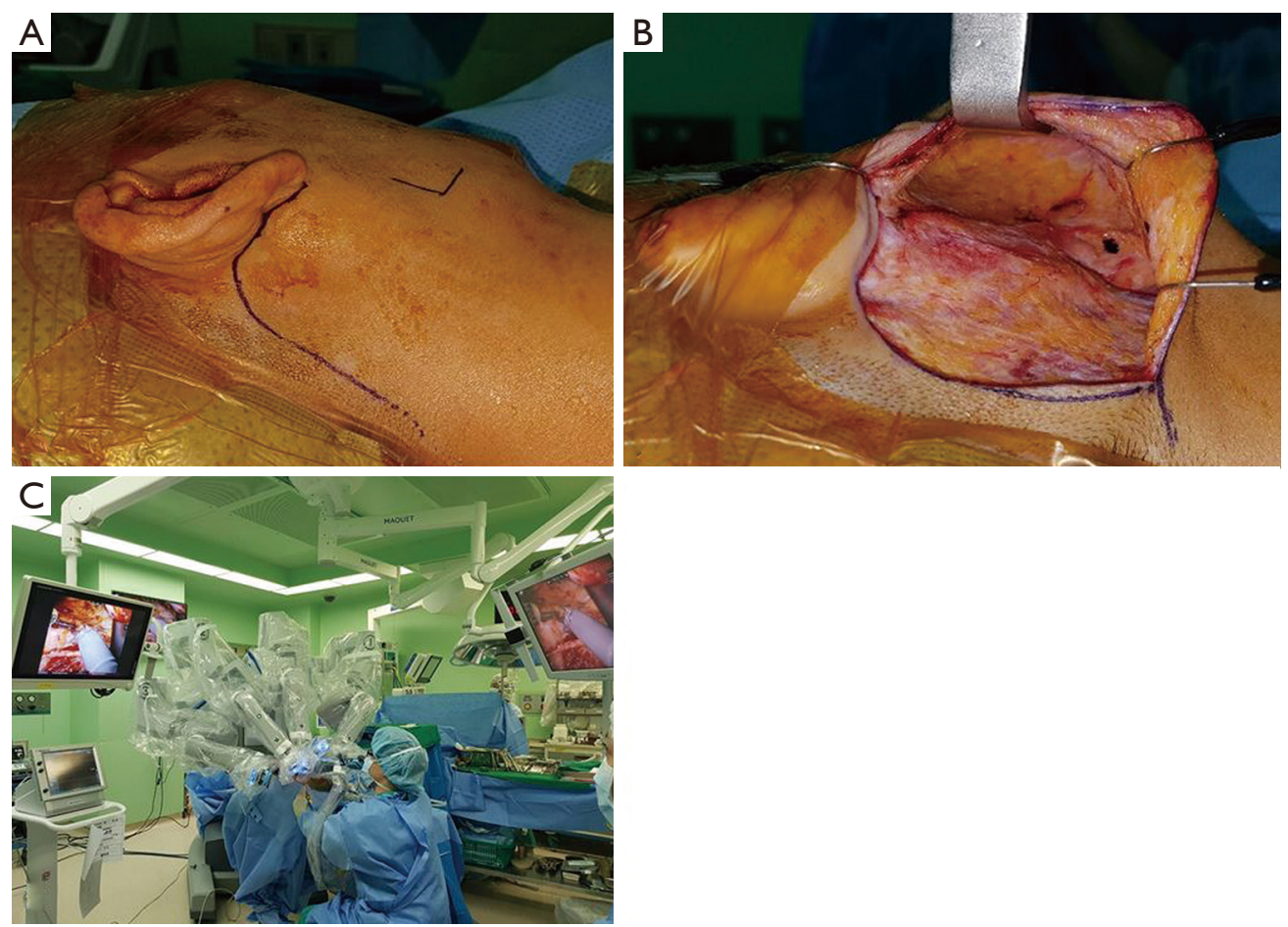

Figure 1 Robotic parotidectomy via a retroauricular incision. (A) Design of retroauricular skin incision; (B) after skin flap was elevation, the elevated skin flap was maintained with self-retaining retractor. Then, mandible angle was marked with blue ink and it was used as anatomical land mark during the surgery; (C) robotic surgical system was introduced through the retroauricular incision.

preoperative imaging studies.

\section{Preoperative assessment}

In all patients with parotid tumor, we performed US guided fine needle aspiration biopsy and CT scan preoperatively. In suspected cases of malignant parotid tumor, PET and MRI were performed additionally to evaluate the extent of the disease and distant metastasis.

\section{Operative procedure}

All surgeries were performed by senior author YW Koh and employed the da Vinci robotic system Xi (Intuitive Surgical Inc., Sunnyvale, CA, USA). Under general anesthesia, the patient was placed in the supine position on a surgical bed, a shoulder roll was placed under the shoulder, and the head was slightly rotated to the side opposite the lesion as is the case in conventional parotidectomy. After designing the retroauricular incision, the flap was lifted along the parotico-masseteric fascia. The skin flap was elevated to the zygomatic arch superiorly, the mandible inferiorly, and the anterior border of the parotid gland. Two assistants pulled the flap up using an Army-Navy retractor and a right-angle retractor, while the surgeon raised the skin flap by counter traction of the parotid tissue using Yankauer suction and Cushing forceps. After the skin flap was elevated, the mandible angle was marked with marking pen and used as an anatomical marker during robotic surgery (Figure 1A). Then, a self-retained retractor (Sejong Medical Corporation, Korea) was inserted into this space to secure a working space (Figure 1B). Finally, the robot was docked (Figure 1C).

The following procedure for performing robotic parotidectomy was similar to a conventional parotid surgery technique (tunnel technique). All procedures were conducted using only robotic instruments, such as Maryland forceps, Scissor, and a Cadiere forceps. First, the parotid and the anterior border of the sternocleidomastoid muscle were separated, and the posterior belly of the digastric muscle was identified in the lower edge of the parotid tissue. The great auricular nerve usually divided into two branches and distributed to pre- and peri-auricular area. If the location and extent of the tumor allowed us to preserve the posterior 
branch of a greater auricular nerve, we tried to preserve it. However, if not, we sacrifice it. Dissection was performed along the auricular cartilage, and the tragal pointer was located along the contour of the auricular cartilage. Using the posterior belly and tragal pointer as anatomical guidelines, we identified the main trunk of the facial nerve at $1 \mathrm{~cm}$ inferior to the tragal pointer and at $1 \mathrm{~cm}$ superior to the posterior belly of the digastric muscle. The parotid tissue was excised along the facial nerve through the tunnel technique using scissors mounted on the right arm and a Maryland dissector mounted on the left arm. Based on the location and extent of the tumor, the appropriate resection range was determined. Using the monopolar cautery function (Erbe system) of the scissor mounted on the right arm, bleeding could be minimized during parotid tissue resection. We did not reconstruct superficial parotidectomy defect with allogenic material or rotational flap to restore tissue defect or prevent Frey syndrome. After operation, the specimen was removed through a retroauricular incision, one drain was placed behind the incision line, and the operation was terminated (Figure 2).

\section{Outcome assessment}

Patient personal information, hospital records, operation records, complications, operation time, and amount of bleeding were collected and analyzed. Patient satisfaction with the cosmetic results was assessed on a graded scale of I (extremely satisfied), II (satisfied), III (average), IV (dissatisfied), and $\mathrm{V}$ (extremely dissatisfied) at 6 months after operation.

\section{Results}

A total of 53 patients participated in this study. Their mean age was 39 years; the patients comprised 25 men and 28 women. All patients underwent robotic parotid surgery due to parotid tumors. Tumors were located in the superficial lobe of the parotid gland in 40 patients, and the remaining 13 tumors were located in the deep lobe of the parotid gland. Six different benign tumor types were found in 35 patients in our study population; 10 different malignant tumor types were noted in 18 patients (Table 2). In terms of the TNM stage, 8 patients were T1, 7 patients $\mathrm{T} 2$, and 3 patients were T3. And 15 patients had N0 and 3 patients had N1. Three patients $(9.7 \%)$ had stage III and $28(90.3 \%)$ stage IV disease. Other patient information is summarized in Table 1. The mean operation time was 226 minutes in patients who underwent only parotidectomy and 375 minutes in patients who underwent parotidectomy with simultaneous robotic neck dissection. The average amount of bleeding was at $23 \mathrm{~mL}$, and the amount of drainage after operation averaged $171 \mathrm{~mL}$. When the amount of drainage output was less than $10 \mathrm{~mL}$ per day, we removed the drain. The average length of hospital stay was 6 days (Table 3).

\section{Learning curve of robotic parotidectomy}

Learning curves were analyzed in cases with only robotic parotidectomy. In the initial four cases, the console time varied greatly from 100-200 minutes, although after five cases, the console time stabilized around 100 minutes (Figure 3). Significant decreases in mean operation time were observed as experience with performing robotic parotidectomy increased.

\section{Complications}

The facial nerve branches were identified and dissected easily using the tunnel technique along the main trunk of the facial nerve under a $10 \times$ magnified view of operative field and two worsted robotic arms. There were no cases of facial nerve injury intraoperatively. During surgery, the superficial temporal artery and retromandibular vein deeply located in the parotid tissue were easily identified and preserved. Therefore, significant bleeding could be avoided without the injury of these vessels. There were no cases of tumor spillage in this study. The mean amount of bleeding was $23 \mathrm{~mL}$, and there were no cases requiring blood transfusion. Postoperative complications were transient facial paralysis in three cases, although all recovered spontaneously, and no permanent paralysis was recorded. No other serious complications occurred in this study.

\section{Discussion}

Traditionally, S-shaped Blair incisions have been used for parotid surgery. This approach is well suited for removal of tumors in all parts of the parotid gland. However, in malignant tumors, the Blair incision must be extended into a cervical skin crease in order to perform neck dissection. Also, the Blair incision can leave scars on the face and neck, which can lead to unsatisfactory cosmetic results. On the contrary, a facelift incision, first described by Jost et al., has the advantage of hiding the postoperative scar, because the preauricular incision is designed inside the 

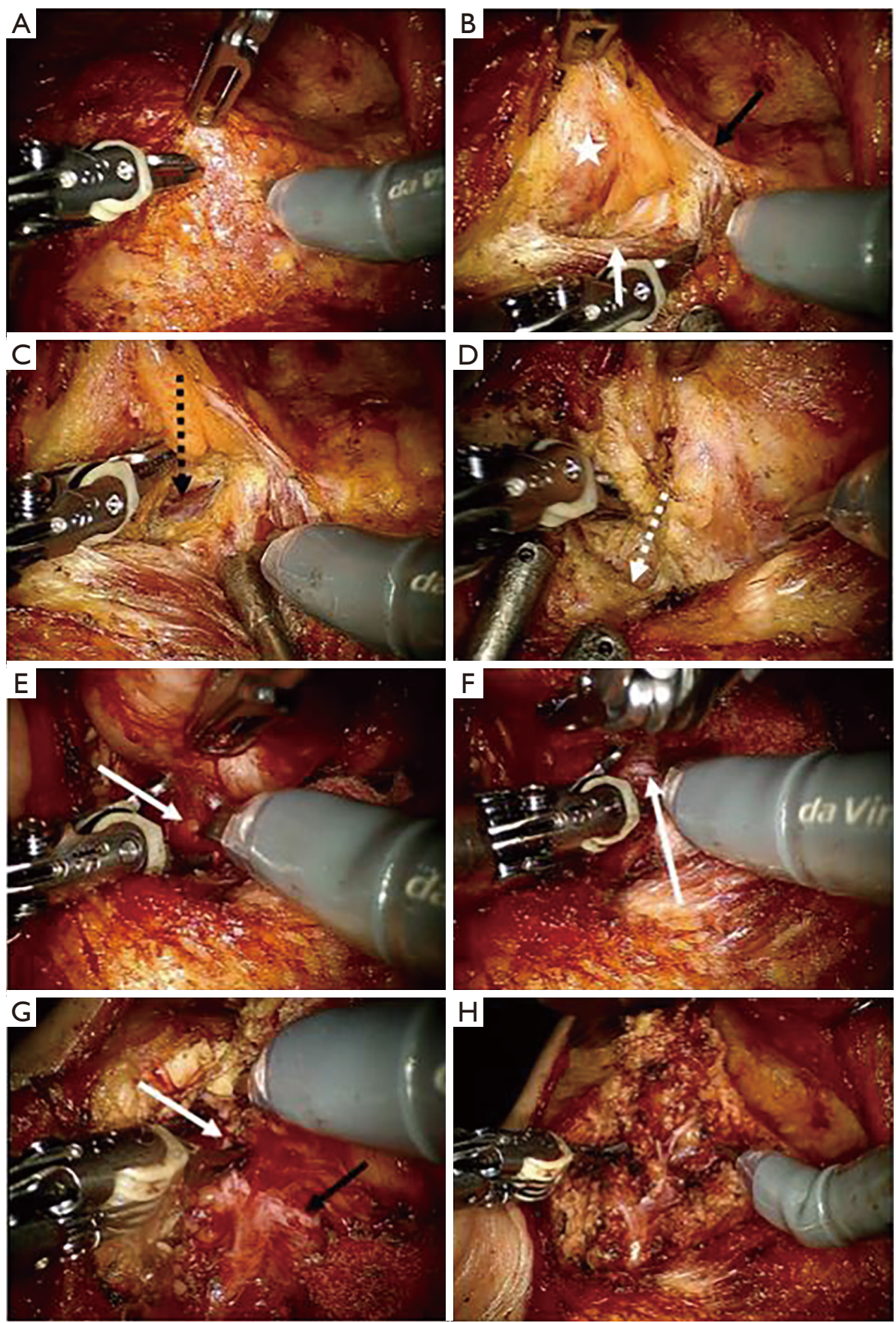

Figure 2 Surgical procedure of robotic parotidectomy on the right side in patients with parotid tumor. (A) Maryland dissector was equipped on left robotic arm and scissor was equipped on right robotic arm. And prograsper forcep was installed on the third robotic arm; (B) the dissection of parotid gland (asterix) was started from the anterior border (white arrow) of sternocleidomastoid muscle (black arrow: greater auricular nerve); (C) posterior belly (black dotted arrow) of the digastric muscle (black arrow) was identified below the inferior part of the parotid gland; (D) a preauricular incision was dissected along the auricular cartilage (white dotted arrow) to find the tragal pointer; (E) the facial nerve main truck (white arrow) was identified at $1 \mathrm{~cm}$ below the tragal pointer and at $1 \mathrm{~cm}$ superior the posterior belly of digastric muscle; (F) dissection was performed along the facial nerve using tunnel technique (white arrow: facial nerve); (G) the superior pole of the parotid gland was dissected along the upper branch (white arrow) of the facial nerve (black arrow: lower branch); (H) after completing the parotidectomy, all branches of the facial nerve were preserved. 
Table 2 Histologic type of salivary diseases in all patients who received robotic parotidectomy

\begin{tabular}{|c|c|c|c|}
\hline & Parotidectomy only ( $\mathrm{n}=36$ ) & $\begin{array}{l}\text { Parotidectomy with robotic neck } \\
\text { dissection }(n=16)\end{array}$ & $\begin{array}{l}\text { Parotidectomy with thyroid } \\
\text { lobectomy }(n=1)\end{array}$ \\
\hline \multicolumn{4}{|l|}{ Malignant tumor } \\
\hline Secretory ca. & 2 & 1 & 0 \\
\hline Salivary duct ca. & 0 & 2 & 0 \\
\hline Acinic cell ca. & 0 & 2 & 0 \\
\hline Squamous cell ca. & 0 & 1 & 0 \\
\hline Myoepithelial ca. & 1 & 0 & 0 \\
\hline Lymphoma & 1 & 0 & 0 \\
\hline Neuroendocrine ca. & 0 & 1 & 0 \\
\hline Pleomorphic adenoma & 22 & 0 & 1 \\
\hline Warthin's tumor & 5 & 0 & 0 \\
\hline Basal cell adenoma & 1 & 0 & 0 \\
\hline Oncocytoma & 1 & 0 & 0 \\
\hline Schwannoma & 1 & 0 & 0 \\
\hline Myoepithelioma & 2 & 0 & 0 \\
\hline
\end{tabular}

Table 3 Perioperative data and postoperative complications

\begin{tabular}{|c|c|c|c|}
\hline & Parotidectomy only $(n=36)$ & $\begin{array}{l}\text { Parotidectomy with neck } \\
\text { dissection }(n=16)\end{array}$ & $\begin{array}{l}\text { Parotidectomy with thyroid } \\
\text { lobectomy }(n=1)\end{array}$ \\
\hline Total operation time, min & 226 & 375 & 256 \\
\hline Estimated blood loss, $\mathrm{mL}$ & 15 & 45 & 10 \\
\hline Drainage, $\mathrm{mL}$ & 133 & 195 & 186 \\
\hline \multicolumn{4}{|l|}{ Complication } \\
\hline $\begin{array}{l}\text { Temporary facial } \\
\text { weakness }\end{array}$ & 2 & 1 & 0 \\
\hline $\begin{array}{l}\text { Permanent facial } \\
\text { weakness }\end{array}$ & 0 & 0 & 0 \\
\hline I & 34 & 15 & 1 \\
\hline ॥ & 2 & 1 & 0 \\
\hline III & 0 & 0 & 0 \\
\hline IV & 0 & 0 & 0 \\
\hline V & 0 & 0 & 0 \\
\hline
\end{tabular}




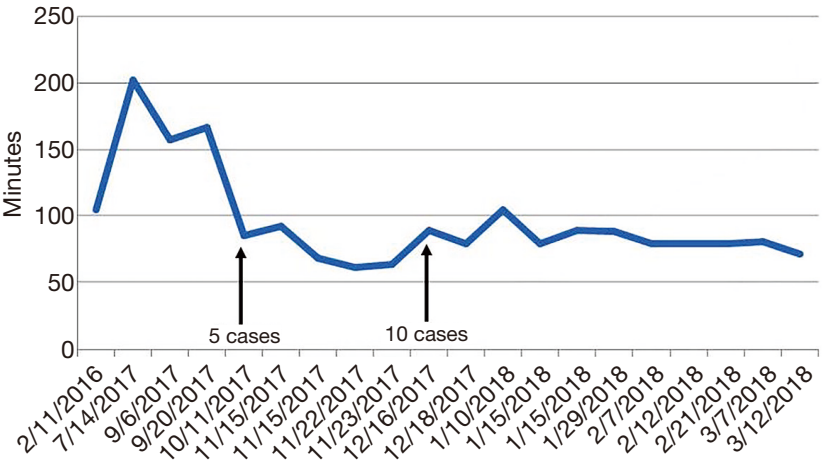

Figure 3 Learning curve of robotic parotidectomy.

tragus (7). Therefore, a facelift incision can improve patient cosmetic satisfaction after operation, but it is somewhat disadvantageous to remove tumors in the superior or anterior parts of the parotid gland because of limited sight $(8,9)$. It is also difficult to apply this method to patients with high-grade malignant tumors who require neck dissection.

Recently, the V-shaped minimal facelift incision reported by some researchers can maximize cosmetic results, because it avoids visible scars on the face and neck and prevents the hypertrophic scar and alopecia problems which can occur in the area of facelift incision (10). However, since extracapsular dissection should be performed for removal of the tumor in cases of $\mathrm{V}$-shaped minimal incision, there is a disadvantage in that this technique can only be applied in very limited cases, depending on location and specific histologic type of parotid tumor (esp. Warthin tumor). Because some parotid gland benign tumors should be removed with sufficient surrounding normal tissue to prevent recurrence of the tumor, and extracapsular dissection is highly likely to lead to remained microscopic tumor tissue, tumor recurrence and facial nerve damage depending on anatomical location of tumor.

Meanwhile, robotic parotidectomy through a retroauricular incision can be applied to remove tumors in all parts of the parotid gland. With our surgical technique, even for malignant tumor, robotic neck dissection can be performed without an extended cervical incision in our surgical technique. Therefore, it is possible to maximize the functional result including cosmetic satisfaction, after operation. Also, with a $10 \times$ magnified view of the operative field in robotic surgery, it is easy to identify and preserve the facial nerve through fine dissection of the parotid tissue using the three robotic arms. In addition, a newly designed detachable nerve monitor could be attached to the robot arm to monitor the integrity of the facial nerve intraoperatively.

Provided with a three dimensional magnified view of operative field with the robotic system, we found it easy to distinguish the surrounding blood vessels and the facial nerve, which may lead to preservation of all branch of the facial nerve after surgery. As the 10 -fold magnified view of the operative field helped the surgeon detect blood flow within the vessels, the surgeon could distinguish it from the facial nerve easily. Also, a facial nerve monitoring system that can be mounted on a robot arm was used in our study; the instrument is mounted on the robot arm can be used like a nerve probe of facial nerve, making it possible to detect the integrity of the facial nerve in real time. In addition, the $10 \times$ magnified visualization and the fine tissue manipulation supported by the robotic system was helpful in identifying the distal branches of the facial nerves, and the two robotic arms could be finely manipulated to carefully dissect the parotid gland in order to minimize bleeding and preserve the nerve. Present endoscopic systems also provide a magnified view with a possible $3 \mathrm{D}$ capabilities. However, when performing endoscopic surgery, a surgical assistant is needed to hold the endoscope beside the surgeon and to adjust the axis and direction of the endoscope interactively according to the operation process. Also, endoscopic instruments are still long and rigid, compared to robotic instruments. The articulated joints of robotic instruments allow surgeons to perform more delicate and precise surgery, compared to endoscopic surgery.

The da Vinci robot system has advanced from the $\mathrm{Si}$ system to $\mathrm{Xi}$ system, although we only used the $\mathrm{Xi}$ system to perform robotic parotidectomy. The $\mathrm{Xi}$ system has been upgraded in several aspects, compared to Si system. Overhead boom rotation allows for multi-dimensional access toward the face and neck without axis limitation. Also, external collisions between the robotic arms in a narrow space is reduced. Finally, the robotic arms of $\mathrm{Xi}$ system can rotate 360 degrees freely in a narrow space, unlike the "snake-like movement" of the Si system. This allows for more precise and delicate dissection of the facial nerve within a confined space.

The newest da Vinci SP system was designed and developed as a single port, flexible robot system and was approved by the FDA for urological surgery in 2014 . Since three robot arms are inserted through a single arm, the motion range of the elbow joint of the robot arm is much smaller than it of the da Vinci Si/Xi system, so it is more suitable for robotic head and neck surgery through a 
remote access port with a narrow and deep working space, such as a retroauricular approach. The working space of a retroauricular approach is an elongated cone shape. As its distal area is deep and narrow, collision between robotic arms can occur in robotic surgery using previous system. As the da Vinci SP system provide free movement within a limited working space, this issue could be resolved, allowing surgeons to perform more comfortable and delicate surgery.

Postoperative sequelae, such as facial nerve dysfunction, facial depression, and gustatory sweating after parotid surgery may affect the quality of life of patients (11-14). As only 55 patients were enrolled in this study and the follow-up period was not enough, it was not possible to generalize our results in the aspect of postoperative complications (15). However, temporary facial paralysis was only observed in three cases, all of which recovered spontaneously, and permanent facial nerve palsy was not observed. Also, postoperative hemorrhage and seroma was not significantly increased with robotic parotidectomy compared to conventional parotid surgery. In our study, most patients were extremely satisfied with the cosmetic results after surgery. In particular, patients who underwent robotic parotidectomy with simultaneous robotic neck dissection were extremely satisfied with their cosmetic results because of the scar hidden below the hairline. Meanwhile, Quiriny et al. reported that a recurrence rate of $1.5 \%$ in patients with benign tumor, although it would be higher in pleomorphic adenoma (16). However, as the average time for disease recurrence after surgery is 10 years, the oncologic safety and long-term outcomes will need to be analyzed in further studies.

\section{Conclusions}

Robotic parotidectomy was found to be a feasible and safe technique in the treatment of parotid tumor. Especially, this operative technique could be helpful in young patients with malignant parotid tumor who should receive cervical lymphadenectomy and parotidectomy, because it does not leave a visible scar on the face or neck. In the future, longterm results regarding the oncologic safety and functional outcome should be evaluated to confirm the clinical relevance of our operative technique in the treatment of parotid cancer.

\section{Acknowledgments}

Funding: This work was supported by the National Research
Foundation of Korea (NRF) grant funded by the Korea government (MSIT) (No. 2018R1C1B6005984).

\section{Footnote}

Conflicts of Interest: All authors have completed the ICMJE uniform disclosure form (available at http://dx.doi. org/10.21037/gs.2020.01.05). The authors have no conflicts of interest to declare.

Ethical Statement: The authors are accountable for all aspects of the work in ensuring that questions related to the accuracy or integrity of any part of the work are appropriately investigated and resolved. The Institutional Review Board of Yonsei University approved this study (No. 4-2019-0765) and waived the requirement for informed consent.

Open Access Statement: This is an Open Access article distributed in accordance with the Creative Commons Attribution-NonCommercial-NoDerivs 4.0 International License (CC BY-NC-ND 4.0), which permits the noncommercial replication and distribution of the article with the strict proviso that no changes or edits are made and the original work is properly cited (including links to both the formal publication through the relevant DOI and the license). See: https://creativecommons.org/licenses/by-nc-nd/4.0/.

\section{References}

1. Kang SW, Jeong JJ, Nam KH, et al. Robot-assisted endoscopic thyroidectomy for thyroid malignancies using a gasless transaxillary approach. J Am Coll Surg 2009;209:e1-7.

2. Kim HY, Chai YJ, Dionigi G, et al. Transoral robotic thyroidectomy: lessons learned from an initial consecutive series of 24 patients. Surg Endosc 2018;32:688-94.

3. Byeon HK, Holsinger FC, Tufano RP, et al. Robotic total thyroidectomy with modified radical neck dissection via unilateral retroauricular approach. Ann Surg Oncol 2014;21:3872-5.

4. Byeon HK, Holsinger FC, Duvvuri U, et al. Recent progress of retroauricular robotic thyroidectomy with the new surgical robotic system. Laryngoscope 2018;128:1730-7.

5. Byeon HK, Kim da H, Chang JW, et al. Comprehensive application of robotic retroauricular thyroidectomy: The evolution of robotic thyroidectomy. Laryngoscope 
2016;126:1952-7.

6. Shin YS, Choi EC, Kim CH, et al. Robot-assisted selective neck dissection combined with facelift parotidectomy in parotid cancer. Head Neck 2014;36:592-5.

7. Jost G, Guenon P, Gentil S. Parotidectomy: a plastic approach. Aesthetic Plast Surg 1999;23:1-4.

8. Kim DY, Park GC, Cho YW, et al. Partial Superficial Parotidectomy via Retroauricular Hairline Incision. Clin Exp Otorhinolaryngol 2014;7:119-22.

9. Woo SH, Park JJ, Kwon M, et al. "Hidden scar" submandibular gland excision using an endoscope-assisted hairline approach. Oral Oncol 2017;65:83-8.

10. Zheng Z, Li J, Wang J, et al. Hidden Scar Dissection of Benign Parotid Gland Tumors via a V-Shaped Minimal Facelift Incision. J Craniofac Surg 2018;29:2299-303.

11. Chaudhary VK, Rawat DS, Tailor M, et al. Post Parotidectomy Quality of Life in Patients with Benign Parotid Neoplasm: A Prospective Study. Indian J Otolaryngol Head Neck Surg 2019;71:363-8.

Cite this article as: Park YM, Kim DH, Kang MS, Lim JY, Kim SH, Choi EC, Koh YW. Real impact of surgical robotic system for precision surgery of parotidectomy: retroauricular parotidectomy using da Vinci surgical system. Gland Surg 2020;9(2):183-191. doi: 10.21037/gs.2020.01.05
12. Lin HJ, Hsiao JR, Chang JS, et al. Resected specimen size: A reliable predictor of severe Frey syndrome after parotidectomy. Head Neck 2019;41:2285-90.

13. Fiacchini G, Cerchiai N, Tricò D, et al. Frey Syndrome, First Bite Syndrome, great auricular nerve morbidity, and quality of life following parotidectomy. Eur Arch Otorhinolaryngol 2018;275:1893-902.

14. Kaya BV, Kılıç C, Özlügedik S, et al. Long-term effects of parotidectomy. Eur Arch Otorhinolaryngol. 2016;273:4579-83.

15. Stathopoulos P, Igoumenakis D, Smith WP. Partial Superficial, Superficial, and Total Parotidectomy in the Management of Benign Parotid Gland Tumors: A 10-Year Prospective Study of 205 Patients. J Oral Maxillofac Surg 2018;76:455-9.

16. Quiriny M, Dekeyser C, Moreau M, et al. Benign tumors of the parotid gland: a retrospective study of 339 patients. Acta Chir Belg 2017;117:227-31. 